\title{
AN EFFICIENT APPROACH FOR CONTENT BASED IMAGE RETRIEVAL USING HIERARCHICAL PART-TEMPLATE AND TREE MODELING
}

\author{
Pushpalatha S. Nikkam ${ }^{1}$ and B. Eswara Reddy ${ }^{2}$ \\ ${ }^{I}$ Department of Computer Science Engineering, Jawaharlal Nehru Technological University Anantpur, India \\ ${ }^{2}$ JNTUA College of Engineering, India
}

\begin{abstract}
Image based content recognition and retrieval is critical in many applications. Existing mechanisms for content based image retrieval lack in terms of performance. In this paper a hierarchical template tree based CBIR system is described. Content in image is represented using a combination of shape features and low level features. Comprehensive feature set definitions proposed enables in achieving better performance. Shape and low level features are considered as templates. Templates of similar categories are further decomposed to form a hierarchical template tree. Query image is converted into a query template and is decomposed. A part template based matching scheme and SVM classifier is used to retrieve visually similar images. Results presented in the paper prove superior performance of proposed technique when compared to recent existing mechanisms in place. An improvement of $10.45 \%$ and $9.69 \%$ in mean average precision and mean retrieval accuracy is reported using proposed approach.
\end{abstract}

Keywords:

Part-Template, Hierarchical Template Tree, HOG, Shape, TreeFormation, SVM Classifier

\section{INTRODUCTION}

In recent years, the use of image database increased widely due to its cost effectiveness and easy maintenance. Therefore, it becomes real easy for the users to utilize the millions of images and videos contains in the database through internet. These image or video database are frequently used in the field of medical applications, satellite image processing, biometric security, engineering applications and in research departments. Therefore, there is need to develop techniques which can easily retrieve the query images or videos from those databases. This attracted researchers to invent some new schemes to organize large images or videos and content based data retrieval effectively. CBIR (content based image retrieval) is a technique to retrieve the same type of images from a large database.

An image represents many low level features such as shape, texture and color. However, in an image shape is the most significant and essential feature to describe an image in content based image retrieval (CBIR). Shape feature used to identify the shape of objects. However, this feature alone is insufficient to identify the content accurately. In [7], a 3D representation technique presented to achieve skin lesion classification based on shape, color and texture feature. However, it suggested that shape is insufficient to detect the content efficiently. In [10], a novel Fourier descriptor based on shape feature presented to increase the efficiency of CBIR. However, shape feature can only work efficiently only for contour based approaches, not for region-based approaches. In [14], for visual semantic analysis and discriminative classification a segmentation approach based on shape, color and texture feature presented. However, it generates irregular shapes for certain regions. There are few more problems occurs in image retrieval such as complexity in query image scenes, shadowing issue, illumination issue, nearest neighbor search problem and optimization difficulty etc. Therefore, there is need to incorporate color or texture feature or both the features together with shape feature for the efficient detection of the content.

Therefore, to eliminate these issues a great amount of work has been carried out by the researchers in recent years. Recently, a hashing technique has been presented to deal with nearest neighbor search and complexity issue by producing a compact binary code for large image retrieval and achieving promising outcomes [2], [4-6] for large image retrieval. However, hashing methods make use of only unlabeled data for generating binary code which led to optimization and normalization problem. A compact binary image encoding technique presented to reduce the high dimensional feature problem which can limit the performance and affect the storage capacity. However, the large optimization problem need to resolve which produced while encoding [3]. A graph-based query specific fusion is presented to perform feature level fusion which improves the precision based on their ranks [8]. However, it cannot combine or measure the cues whose effectiveness changes drastically for a large dataset. A Novel Approach to Visual Recognition using sparse spatial coding presented for object recognition by reducing novel approach problem [9]. However, a $L 1$-regularized least squares normalization complexity occurs in this technique.

To overcome this drawbacks this paper discusses a hierarchical template tree matching approach. Images in CBIR database are said to belong to categories. For each category a hierarchical tree is maintained. The CBIR system consists of a training and testing phase. In training phase key point based shape detection from [1] is adopted to construct initial templates. Shape feature extracted depicts content of the training/testing image. Literature presented in [7], [10], [14] prove that shape feature alone is insufficient to accurately define content identified. In addition to shape features low level or finer additional features are extracted using Histogram of oriented gradient (HOG) features. Fine HOG features enable in understanding intensity variations of contents identified while preserving shape features. HOG features are robust to shadowing and illumination effects which render them advantageous. A template with shape and HOG features is obtained in training per training image per category. Templates of a category are decomposed into forming a hierarchical tree. In testing phase, query image represented as a query template (shape + HOG features) is decomposed and block wise matching against template trees is considered in this approach. Support Vector Machines (SVM) is used to obtain visually similar images. A detailed modelling and experimental study considering proposed CBIR is 
presented in further sections of the paper. For performance evaluation popular Corel $5 \mathrm{~K}$ is adopted. Experimental results with comparisons with existing state of art techniques is considered. Superior shape feature extraction performance and CBIR functionality is verified.

This paper is organize in following sections, which are as follows. In section 2, we described our proposed methodology. In section 4, experimental results, evaluation shown, and section 5 concludes our paper. The references used throughout manuscript are given at the end of the paper.

\section{RELATED WORK}

This section describes about the recent work carried out for CBIR (Content based Image Retrieval) using HOG feature. The efficiency of CBIR system can be increased by extracting effective HOG features of an image in cooperation with shape features based on their key points. Fine HOG feature can detect the intensity variations while preserving shape features. In [15], HOG (Histogram of gradients) presented for human detection using IOT services to reduce the problems of view point, fixed resolution. However, this process is little expensive while computing. In [16], a new content based image retrieval technique presented based on approximate shape to measure the similarity between shapes of images. In [17], a content based image retrieval approach presented based on shape, texture and color feature to achieve efficient semantic processing. However, it requires high computational complexity. In [6], compact binary code for large image retrieval based on color feature proposed to for large image retrieval. However, hashing methods make use of only unlabeled data for binary code which led to optimization and normalization problem. In [11], a modified block truncation coding presented based on the texture for CBIR system .In this approach the key idea is to get feature vector from the threshold mean over different non-overlapped blocks. In [12], scalable image retrieval approach presented to coordinate smartly with huge histo-pathological images. Here, a supervised kernel hashing approach discussed to reduce the semantic gap between low-level and high-level features. In [13], to identify the background and object regions dual multiscale gray level morphological open technique presented. To deal with large scale datasets SEGON approach incorporated with morphological open technique. However, in this approach boundary cut problem occurs and to deal with that an additional technique incorporated which only increases the computational overhead.

\section{PROPOSED MODEL}

For efficient CBIR system we propose hierarchical approach for matching the image template and combine it with the learning process to build detector architecture for the CBIR system. The CBIR system considers visually similar images (i.e. images of similar types, like beach, bus, dinosaur etc.) to belong to a specific category. According to the proposed model a query image is given as an input which returns a query template. A query template consists of the shape detected and the internal low level features within detected shape. Query template is matched against a template set created during training. During matching, global and local templates are decomposed to construct a part-template tree based on the shapes and its corresponding low level/fine features. Query template is split into grids and is matched with the template tree constructed to determine the efficiency of CBIR system.

During training phase, for each image per category, shape detected and the internal features within detected shape is extracted and is referred to as training template. The training templates per category are combined to construct a global tree structure. Template tree structure is a hierarchical representation of decomposed part-training templates. In other words a training template is split into parts and each part represents a node in the global tree structure of a category. For feature extraction or detected shape description representation we consider the HOG features. This approach gives the better spatial features for local shape data.

\subsection{TEMPLATE CONSTRUCTION USING SHAPE AND INTERNAL FEATURE DESCRIPTORS}

In the initial step, shape of the content present in the image is extracted. In proposed CBIR system shape is extracted using method described in [1]. Let us consider an image $I$, its shape feature at location $l=(x, y)$ according to [1] is defined as,

$$
K^{l}(J, I)=\bigcup_{i=1}^{N} S^{l_{i}}\left(J, P_{i}\right)
$$

where, detection window is $J$, operator $U$ represents redundant union on subsets, $N$ is number of sub-templates and $S^{l_{i}}\left(J, P_{i}\right)$ represents a set of key points used to describe shape of the $i^{\text {th }}$ section of the template at position $l_{i}$.

On obtaining shape of the concept, its internal features need to be considered for higher accuracy of retrieval. In proposed CBIR system HOG features are considered to represent shape internal parameters. To extract internal features, gradient magnitude of $I$ (represented by $\left|G_{m a g}\right|$ ) and the edge-orientation $\varepsilon_{o}$ in both $x, y$ (or horizontal and vertical) directions is computed by using simple difference operators. Difference operators are represented as $(-1$, $0,1),(-1,0,1)^{t} . I$ is quantized into $8 \times 8$ non-overlapping blocks which are represented by the histogram and edge orientation. Similarly, edge orientation of $I$ is quantized into various orientation bins which is given by,

$$
O_{\text {bins }}=\left[k\left(\frac{\pi}{O_{\text {bins }}}\right),(k+1)\left(\frac{\pi}{O_{\text {bins }}}\right)\right]
$$

where $k=0,1,2,3,4, \ldots, O_{\text {bins }}$.

Improving spatial and orientation dimensions is achieved by eliminating discontinuity and aliasing effects. These effects are eliminated using trilinear interpolation, where each cell forms a $2 \times 2$ block for the image representation. Gradient of $I$ in $x$ direction, $y$-direction is given by,

$$
\begin{aligned}
& G_{x}=\frac{\delta I}{\delta x}=f(x+1, y)-f(x-1, y) \\
& G_{y}=\frac{\delta I}{\delta y}=f(x, y+1)-f(x, y-1)
\end{aligned}
$$

where, $G_{x}, G_{y}$ denotes the gradient representation in $x, y$ direction, $x$ and $y$ shows the direction, horizontally and vertically. Consequently magnitude of the gradient and orientation is computed using, 


$$
\begin{gathered}
\text { GradMag : } G_{\text {mag }}(x, y)=\left(G_{x}^{2}+G_{y}^{2}\right)^{0.5} \\
\text { GradOrient }: G_{\text {orient }}(x, y)=\tan ^{-1}\left(\frac{G_{x}}{G_{y}}\right)
\end{gathered}
$$

Post computation of $G_{m a g}(x, y), G_{\text {orient }}(x, y)$ the $L 2$ Normalization on the data is considered given by,

$$
\text { L2-Norm: }\left\{\left(\frac{c}{\left\|B_{k}\right\|^{2}+\varepsilon^{2}}\right)^{0.5}\right\}
$$

where, $\varepsilon$ is the regularization constant, $B_{k}$ represents the blocks of the image.

Accumulating histograms of all overlapping blocks in detection window is $J$ of $I$ provides the shape internal descriptors represented as $S=\left\{s_{1}, s_{2}, s_{3}, \ldots, s_{\text {Obins }}\right\}$, where $O_{\text {bins }}$ is total number of bins. The shape and corresponding internal features are considered as template of $I$ and are referred to as shape features for ease of representation. The shape and partial set of templates constructed using dinosaur and flower images from dataset [3] is shown in Fig.1.
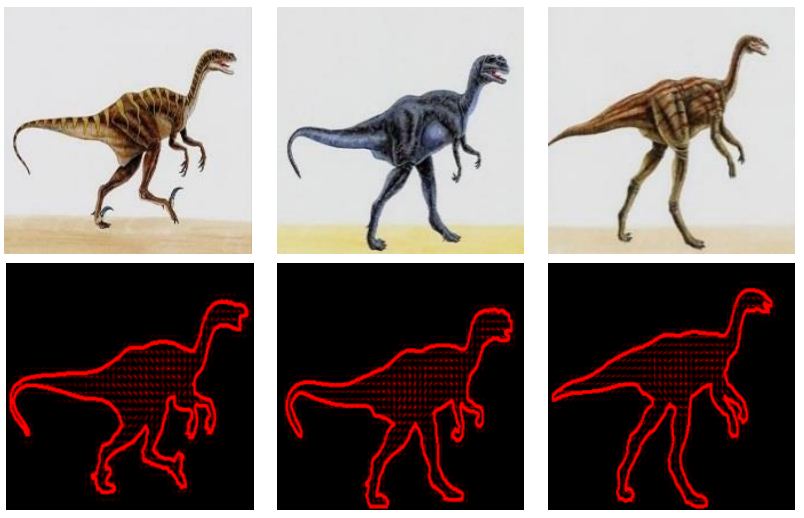

(a) Sample images and templates considering Dinosaur images from dataset
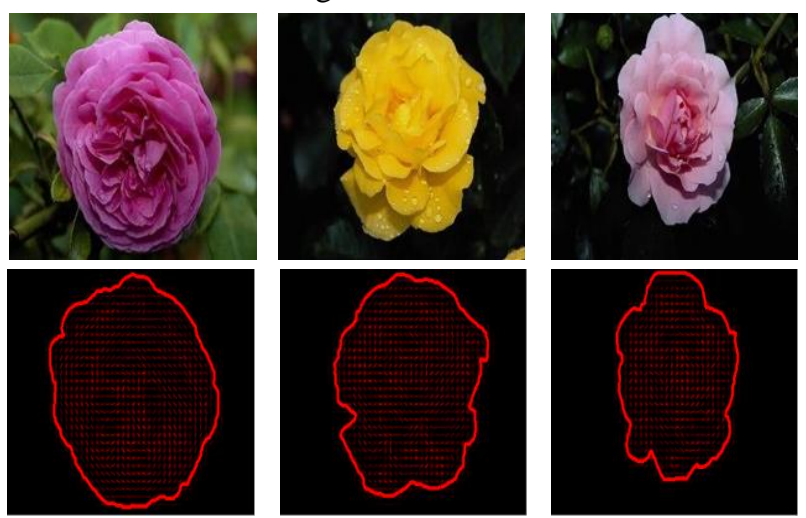

(b) Sample images and templates considering flower images from dataset

Fig.1. Sample templates constructed using shape and encompassing internal feature

\subsection{TREE MODEL CONSTRUCTION FOR PART- TEMPLATE MATCHING}

For each category a tree model is constructed using all training shape templates of that category. Each shape template is decomposed into part templates that cumulatively describe complete shape feature. A tree construction with part templates enables in accurate matching and is useful to handle shape deformation, size variations, spatial differences and inaccurate or partial shape identifications in CBIR systems. For example in presence of complex backgrounds, cluttered images complete shape identification is difficult and only partial shape is obtained, in such cases part templates of the tree is useful for CBIR applications.

To overcome complexity of shape tree representation we propose dual stage learning method. According to this method a global shape model is generated initially, and then it is decomposed to form a part-template hierarchy used in tree construction. This part template is generated by decomposing the total shape feature and part-template tree is achieved. The tree constructed by decomposing all shape models of training images contains all the variation of the objects defining a category. This model of template tree is refined by learning from the small set training images. A sample shape template construction of a horse image using sub templates is shown in Fig.2.

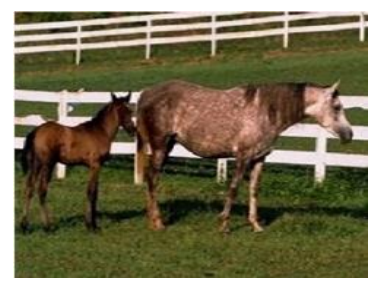

(a)

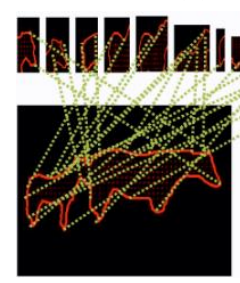

(b)

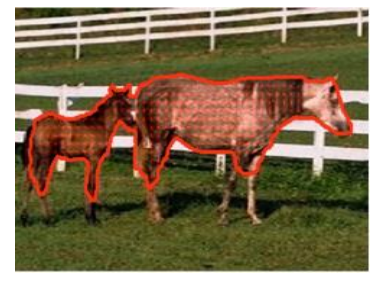

(c)

Fig.2. (a) Original image, (b) Shape template construction using part templates, (c) Horse image with shape and shape internal features identified

Learning process is performed during training stage of the CBIR system. During this process each training image is passed through the tree structures using root node and leaf node. Using this approach shape coverage is observed. Each part template is said to cover area $P$ of total shape feature image. The pixel based similarity between the $r^{\text {th }}$ part template tree of training image i.e. 
$Q_{r_{p}}$ and corresponding part template of object tree $T_{p}$ is defined as,

$$
\alpha\left(r_{p} \mid T\right)=\left(1-\left(\frac{\sum_{x}\left|T_{p}(x)-P\left(r_{p}, x\right)\right|}{n}\right)\right)
$$

where $n$ is the number of pixels in the shape coverage area. As template tree consists of multiple part templates, matching query template with part templates of tree $T$ provides numerous matching results. Best matching part templates of tree $T$ is represented using set $r=\left\{r_{p}{ }^{\prime}\right\}$ is obtained using,

$$
\left\{r_{p}^{\prime}\right\}=\arg \max _{r_{p} \in S_{p}} \alpha\left(r_{p} \mid T\right)
$$

where $S_{p}$ represents a set of all shape feature parameters i.e. shape and corresponding internal features. The matching score of a data point on the part-layout form is measured by edge, shape, HOG features and orientation matching.

This process is repeated for all training images and the ranges of part template models are estimated based on the statistics of each part-template's model parameters. The ranges of parameters are evenly quantized to produce the final tree model.

A detection window based shape extraction and feature matching is adopted here. In the detection window the overall matching score is computed as summation of all matching values. Testing phase is performed on the shape, edge and orientation of the dataset and matching score is computed for each node in the detection window. The part template matching enables in complete template matching (as part templates are a subset of the complete template). The main objective of part template matching is to evaluate the shape, which is most reliable with the image perceptions.

In the testing phase of proposed CBIR system, shape template of query image is extracted. The template of the query image is decomposed into part templates and then matched against various trees using pixel based similarity defined earlier. (For each category trained a template tree is constructed in training. As the system is trained for multiple category images multiple training trees are generated). A block wise part template is considered. In block wise matching gradient of the data, shape, edge and orientations is calculated. For each template the computation of score is performed by using key point based lookups. There are some noises in the orientation which is diminished with the help of magnitudes of neighboring histograms. This helps to improve the matching score.

Let $O_{e}(t)$ denote the orientation of object at the $t^{\text {th }}$ point of the shape template. Its corresponding orientation bin index represented as $N(t)$ is computed using,

$$
N(t)=\frac{O_{e}(t)}{\left(\pi / O_{\text {bins }}\right)}
$$

For each part template a detection window of $z \times z$ is considered. The matching score of the detection window at the $t^{\text {th }}$ point of the shape template is computed using,

$$
M(t)=\sum_{k=-\delta}^{\delta} W(k) s_{N(t)+k}
$$

where $W(k)$ is the weight distribution, $\delta$ is the range of neighbor pixels considered and $s_{(N(t)+k)} \in S$ is the shape and its encompassing internal features of the $(N(t)+k)^{\text {th }}$ bin. Using Eq.(11), the matching score of $T^{\text {th }}$ part template consisting of $|S|$ is the number of sample key data points is,

$$
M(T)=\frac{1}{|S|} \sum_{t \in S} M(t)
$$

Using the part template matching score the template matching score is computed for every query image. Proposed CBIR model considers SVM to identify category and retrieve visually similar images. Experiments undertaken to evaluate performance of CBIR system is discussed in the next section of paper. Extracted shape feature vectors of the query image is used by SVM to identify category or class and retrieve visually similar images within the identified class. The performance of the proposed shape based CBIR system is evaluated through an experimental study discussed in the next section.

\section{PERFORMANCE EVALUATION}

The proposed CBIR system is developed using Matlab and $\mathrm{C}++$ codes on the Open $\mathrm{CV}$ platform. All experiments are conducted on a machine housing a quad core Intel 17 processor with $16 \mathrm{~GB}$ of RAM. Experiments are conducted to study shape feature extraction performance, shape with low level feature extraction performance and CBIR performance. For evaluation popular Corel $5 \mathrm{~K}$ dataset is considered [3]. The Corel $5 \mathrm{~K}$ dataset contains 100 images per category. The number of categories in Corel $5 \mathrm{~K}$ dataset is 50 . Training and testing databases are constructed according to method described in [9] for uniformity of comparison. In training phase, 50 hierarchical template trees are constructed using training images. Templates created are stored for further comparisons and matching with template queries. The SVM classifier is adopted in proposed CBIR system to retrieve visually similar images.

Performance of CBIR system described in this paper depends entirely on accurate hierarchical template tree construction. The initial step of template tree construction is to identify shape of content defined by Eq.(1). In our earlier work [1] a key point based shape feature extraction method is described. Performance of our shape detector is compared with state of art methods described "SEGON" [13]. The comparison results of SEGON and proposed Shape Feature extraction method is shown in Fig.3. For comparison with SEGON image dataset used in [13] is considered in experiments. From Fig.3 it is evident that proposed key point based shape feature extraction technique exhibits better performance when compared to SEGON.

In proposed CBIR system apart from shape HOG descriptors are adopted to define low level features of objects identified. A holistic feature description enables in improving template matching and CBIR performance. Proposed feature extraction is compared with a recent technique LTxXORP [19]. For comparison of complete feature extraction, a rose image from Corel dataset is considered. Features extracted using LTxXORP and proposed technique is shown in Fig.4. For visual clarity proposed features extracted (i.e. shape and HOG) are represented using RED color. Considering LTxXORP, accurate shape feature definitions is observed. The LTXXORP lacks as it considers 
binary patterns for feature extraction resulting in multiple unrelated feature definitions. Unrelated features obtained using LTXXORP is visible in regions outside the yellow rose. Using proposed technique accurate shape, texture variations with edge preserving features is obtained. Results presented in Fig. 4 prove that proposed feature extraction technique is robust and outperforms existing LTXXORP feature extraction technique.

CBIR performance of proposed technique is evaluated using image retrieval accuracy and retrieval precision described in [8]. The retrieved images using proposed technique for 3 sample queries belonging to dinosaur, castles and art categories is shown in Fig.5. Only the top 5 visually similar images retrieved is shown in the Fig.5. Visually similar images retrieved belong to the same category. Each image of database is either considered for training or testing. This is considered as no overlap in image occurs and testing is in accordance to method described in [9]. Results obtained prove the applicability of proposed hierarchical template tree technique for CBIR applications.

Performance of proposed CBIR system is evaluated using all images in test database. The retrieval precision and retrieval accuracy values are noted. Researchers in [8], [14], [20], [21] and [22] consider mean average retrieval precision $(m A P)$ as a measure of performance. Average retrieval precision of proposed technique is compared with MSD [20], SSH [21], ASD [22], AIA [14] and RANK FUSION [8] techniques. All comparisons consider Corel 5K dataset. In [14] researchers have evaluated AIA technique over a set of experiments with various configuration. For comparison average and best precision is presented here. Results for mAP obtained considering proposed technique and MSD [20], SSH [21], ASD [22], AIA [14], RANK FUSION [8] systems is shown in Table.1. Considering proposed technique $m A P \%$ improvement of $36.64 \%, 33.56 \%, 32.58 \%, 30.99 \%$, $18.03 \%$ is reported compared to MSD [20], SSH [21], ASD [22], AIA (Mean) [14] and AIA (Best) [14]. Best mAP performance among existing technique is reported in [8]. Results presented prove that proposed technique exhibits a higher mAP\% of 10.45 than [8]. Results presented prove best mAP performance when compared to recent image retrieval techniques.
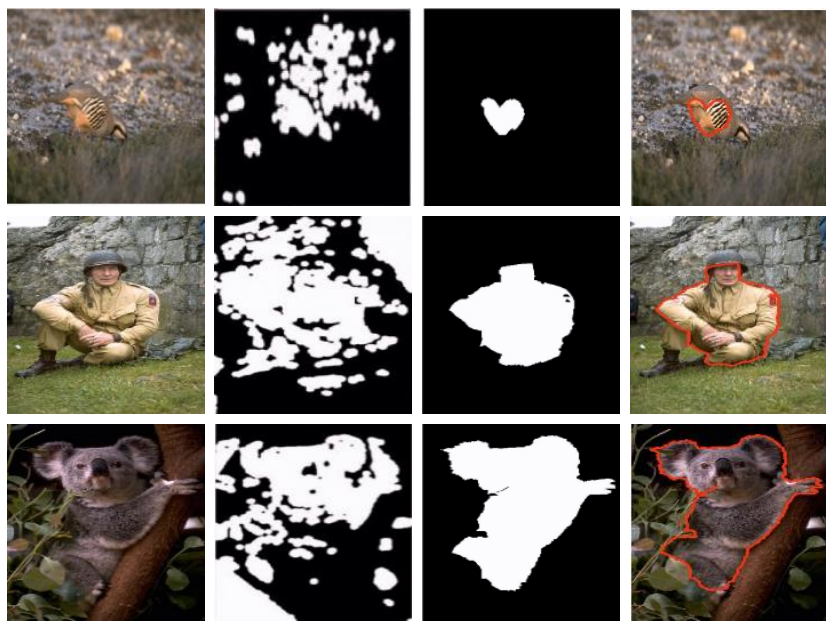

Fig.3. Original input Image, Shape featured by SEGON [13], shape feature using proposed technique and Shape feature identified with original image using proposed key point based shape feature extraction technique
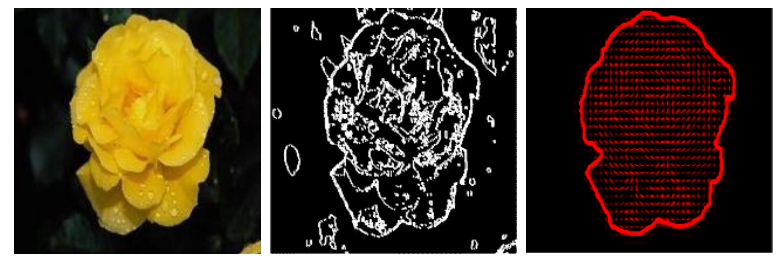

Fig.4. Input Image, Feature extracted using LTxXORP and feature extracted using proposed technique

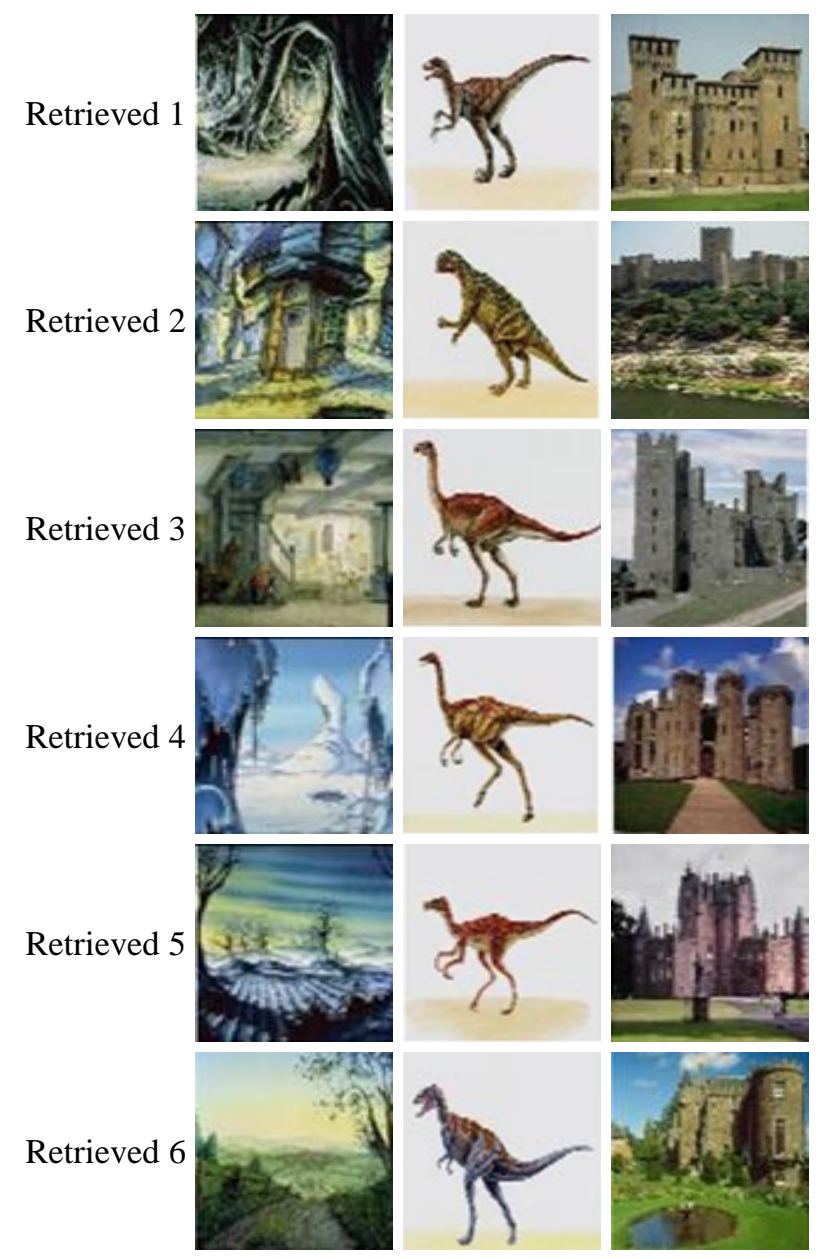

Fig.5. Three sets of visually similar image retrieval results obtained using proposed CBIR technique for sample query images of art, dinosaur and castle

Table.1. Comparison of mAP (\%) on Corel 5K dataset recent image retrieval methods and proposed technique

\begin{tabular}{|c|c|}
\hline Different Methods & $\boldsymbol{m A P}(\boldsymbol{\%})$ \\
\hline MSD [20] & 58.45 \\
\hline SSH [21] & 61.53 \\
\hline ASD [22] & 62.51 \\
\hline AIA (MEAN) [14] & 64.10 \\
\hline AIA (BEST) [14] & 77.06 \\
\hline Rank Fusion [8] & 84.64 \\
\hline Proposed & 95.09 \\
\hline
\end{tabular}


Authors in [8], [9], [23] and [18] consider retrieval accuracy $(r A)$ as a performance measure. Comparison of proposed technique is considered with LLC [23], ScSPM [24], SSC [9] and RANK FUSION [8] recent image retrieval techniques. Considering Corel-5K dataset, results obtained for $r A$ is shown in Table.2. Results obtained prove that proposed CBIR technique exhibits best $r A$ of $94.25 \%$. Improvement in $r A \%$ of $17.23 \%, 16.65 \%, 15.45 \%$ and $9.69 \%$ is reported on comparing proposed technique with LLC [23], ScSPM [24], SSC [9] and Rank Fusion [8].

Table.2. Comparison of $m A(\%)$ on Corel $5 \mathrm{~K}$ dataset recent image retrieval methods and proposed technique

\begin{tabular}{|c|c|}
\hline Different Methods & $\boldsymbol{m A ( \% )}$ \\
\hline LLC [23] & 77.02 \\
\hline ScSPM [24] & 77.60 \\
\hline SSC [9] & 78.80 \\
\hline Rank Fusion [8] & 84.56 \\
\hline Proposed & 94.25 \\
\hline
\end{tabular}

Results presented in the performance evaluation experiments prove that proposed hierarchical template tree CBIR system exhibits robust shape recognition/extraction capabilities. The shape and HOG features considered to construct hierarchical template trees enable accurate representation of content identified in images. The CBIR performance of proposed technique is superior when compared to existing state of art techniques.

\section{CONCLUSION AND FUTURE WORK}

The significance of image retrieval in various applications is discussed. Retrieval is achieved by understanding content within images. Significance of shape and additional low level features to enable retrieval of visually similar images is discussed. A hierarchical template tree based CBIR approach is discussed in this paper. A key point based shape extraction algorithm is considered for content identification. In addition to shape features, content definition is enhanced using HOG for low level feature representation. Content identified is considered as a template. A hierarchical template tree is constructed by decomposing templates of similar categories. For retrieval tasks hierarchical template tree part matching with SVM mechanism is adopted in proposed technique. Results presented prove improved accuracy of content shape identification, comprehensive/robust content feature definitions when compared to existing techniques. Mean retrieval precision improvement of $10.45 \%$ is reported using proposed CBIR technique when compared to rank fusion. An improvement of $9.69 \%$ in terms of retrieval accuracy is also proved. Proposed hierarchical template tree based CBIR technique is robust and exhibits best performance when compared to existing mechanisms in place. Future of work presented here is to evaluate performance of proposed technique considering additional machine learning algorithms and datasets. Applicability of proposed work to incorporate image annotation is a possible future direction.

\section{REFERENCES}

[1] P.S. Nikkam and E.B. Reddy, "A Key Point Selection Shape Technique for Content based Image Retrieval System", International Journal of Computer Vision and Image Processing, Vol. 6, No. 2, pp. 54-70, 2016.

[2] A. Gionis, P. Indyk and R. Motwani, "Similarity Search in High Dimensions via Hashing", Proceedings of International Conference on Very Large Data Bases, pp. 518-529, 1999.

[3] P. Duygulu., K. Barnard, J. de Freitas and D. Forsyth, "Object Recognition as Machine Translation: Learning a Lexicon for a Fixed Image Vocabulary", Proceedings of $7^{\text {th }}$ European Conference on Computer Vision, Vol. 4, pp. 97 112, 2002.

[4] A. Andoni and P. Indyk, "Near-Optimal Hashing Algorithms for Approximate Nearest Neighbor in High Dimensions", Communication of the ACM, Vol. 51, No. 1, pp. 117-122, 2008.

[5] Y. Weiss, A. Torralba and R. Fergus, "Spectral Hashing", Proceedings of Advances in Neural Information Processing System, pp. 1753-1760, 2008.

[6] M. Kan, D. Xu, S. Shan and X. Chen, "Semisupervised Hashing via Kernel Hyperplane Learning for Scalable Image Search", IEEE Transactions on Circuits and Systems for Video Technology, Vol. 24, No. 4, pp. 704-713, 2014.

[7] T.Y. Satheesha, D. Satyanarayana, M.N.G. Prasad and K.D. Dhruve, "Melanoma Is Skin Deep: A 3D Reconstruction Technique for Computerized Dermoscopic Skin Lesion Classification", IEEE Journal of Translational Engineering in Health and Medicine, Vol. 5, pp. 1-17, 2017.

[8] S. Zhang, M. Yang, T. Cour, K. Yu and D.N. Metaxas, "Query Specific Rank Fusion for Image Retrieval", IEEE Transactions on Pattern Analysis and Machine Intelligence, Vol. 37, No. 4, pp. 803-815, 2015.

[9] G.L. Oliveira, E.R. Nascimento, A.W. Vieira and M.F.M. Campos, "Sparse Spatial Coding: A Novel Approach to Visual Recognition", IEEE Transactions on Image Processing, Vol. 23, No. 6, pp. 2719-2731, 2014.

[10] E. Sokic and S. Konjicija, "Novel Fourier Descriptor based on Complex Coordinates Shape Signature", Proceedings of $12^{\text {th }}$ International Workshop on Content-Based Multimedia Indexing, pp. 1-4, 2014.

[11] J.M. Guo, H. Prasetyo and J.H. Chen, "Content-Based Image Retrieval using Error Diffusion Block Truncation Coding Features", IEEE Transactions on Circuits and Systems for Video Technology, Vol. 25, No. 3, pp. 466-481, 2015.

[12] X. Zhang, W. Liu, M. Dundar, S. Badve and S. Zhang, "Towards Large-Scale Histopathological Image Analysis: Hashing-based Image Retrieval", IEEE Transactions on Medical Imaging, Vol. 34, No. 2, pp. 496-506, 2015.

[13] J.J. Chen, C.R. Su, W.E. L. Grimson, J.L. Liu and D.H. Shiue, "Object Segmentation of Database Images by Dual Multiscale Morphological Reconstructions and Retrieval Applications", IEEE Transactions on Image Processing, Vol. 21, No. 2, pp. 828-843, 2012.

[14] J. Zhang, Y. Gao, S. Feng, Y. Yuan and C.H. Lee, "Automatic Image Region Annotation through Segmentation based Visual Semantic Analysis and 
Discriminative Classification", Proceedings of IEEE International Conference on Acoustics, Speech and Signal Processing, pp. 1956-1960, 2016.

[15] D. Sangeetha and P. Deepa, "Efficient Scale Invariant Human Detection using Histogram of Oriented Gradients for IoT Services", Proceedings of $30^{\text {th }}$ International Conference on VLSI Design, pp. 61-66, 2017.

[16] S. Deniziak and T. Michno, "Content based Image Retrieval using Query by Approximate Shape", Proceedings of Federated Conference on Computer Science and Information Systems, pp. 807-816, 2016.

[17] A. Anandh, K. Mala and S. Suganya, "Content based Image Retrieval System based on Semantic Information using Color, Texture and Shape Features", Proceedings of International Conference on Computing Technologies and Intelligent Data Engineering, pp. 1-8, 2016.

[18] J. Yang, K. Yu, Y. Gong and T. Huang, "Linear Spatial Pyramid Matching using Sparse Coding for Image Classification", Proceedings of IEEE Conference on
Computer Vision and Pattern Recognition, pp. 1794-1801, 2009.

[19] A Bala and T Kaur, "Local Texton XOR Patterns: A New Feature Descriptor for content-based Image Retrieval", International Journal on Engineering Science and Technology, Vol. 19, pp. 101-112, 2016.

[20] G.H. Liu and J.Y. Yang, "Content-based Image Retrieval using Color Difference Histogram", Pattern Recognition, Vol. 46, No. 1, pp. 188-198, 2013.

[21] G.H Liu, J.Y Yang, Z.Y. Li, "Content-based Image Retrieval using Computational Visual Attention Model", Pattern Recognition, Vol. 48, No. 8, pp. 2554-2566, 2015.

[22] M. Zhao, H. Zhang and L. Meng, "An Angle Structure Descriptor for Image Retrieval", China Communications, Vol. 13, No. 8, pp. 222-230, 2016.

[23] J. Wang, J. Yang, K. Yu, F. Lv, T. Huang and Y. Gong, "Locality Constrained Linear Coding for Image Classification", Proceedings of IEEE Conference on Computer Vision and Pattern Recognition, pp. 3360-3367, 2010. 